\title{
SEQÜESTRO BRONCOPULMONAR INTRALOBAR: RELATO DE CASO*
}

\author{
Marcelo Souto Nacif ${ }^{1}$, Heliantho de Siqueira Lima Filho ${ }^{2}$, Ricardo Andrade Fernandes de Mello ${ }^{3}$, \\ Gustavo Federico Jauregui ${ }^{4}$, Bruno José de Pinho Miranda ${ }^{5}$, Juliana Mauro Caramel ${ }^{6}$, Alair \\ Augusto Sarmet Moreira Damas dos Santos ${ }^{7}$
}

\begin{abstract}
Resumo Neste trabalho é descrito um caso de uma paciente de 49 anos de idade que apresentava infecções pulmonares de repetição. Na telerradiografia de tórax observou-se massa no segmento basal posterior do pulmão direito, que na angiotomografia e nas reconstruções em 3D notou-se a presença de um suprimento sanguíneo proveniente da artéria aorta descendente. No exame da peça cirúrgica ratificou-se a presença de seqüestro pulmonar intralobar com cavidade preenchida por muco.

Unitermos: Seqüestro pulmonar; Massa pulmonar; Radiologia.
\end{abstract}

Abstract Intralobar pulmonary sequestration: a case report.

We report the case of a 49-year-old patient with repeated lung infections. Chest $x$-rays showed a mass in the posterior basal segment of the right lung. Angiotomography and 3D reconstructions showed a blood supply coming from the descending aorta. The analysis of the surgical specimen confirmed the occurrence of intralobar pulmonary sequestration with a cavitation filled with mucus.

Key words: Pulmonary sequestration; Pulmonary mass; Radiology.

\section{INTRODUÇÃO}

Seqüestro broncopulmonar (SB) é definido como uma malformação congênita na qual uma porção de tecido pulmonar não apresenta comunicação com a árvore brônquica normal, cujo suprimento sanguíneo se faz por uma artéria sistêmica anômala, originada da aorta ou, menos freqüentemente, de seus ramos. A drenagem

* Trabalho realizado no Instituto de Pós-Graduação Médica Carlos Chagas (IPGMCC), Niterói, RJ.

1. Professor Auxiliar da Disciplina de Radiologia da Faculdade de Medicina de Teresópolis, Centro de Ciências Biomédicas, Fundação Educacional Serra dos Órgãos (FMT-CCBM - FESO) Mestrando do Departamento de Radiologia da Universidade Federal do Rio de Janeiro (UFRJ), Membro da Comissão de Residência Médica da Sociedade Brasileira de Radiologia (SBR) Pós-graduado pelo IPGMCC.

2. Médico Radiologista, Pós-graduado pelo IPGMCC e Medimagem/Hospital da Beneficência Portuguesa de São Paulo.

3. Pós-graduado pelo IPGMCC, Mestre em Radiologia pelo Departamento de Radiologia da UFRJ, Membro Titular do Colégio Brasileiro de Radiologia e Diagnóstico por Imagem (CBR).

4. Pós-graduado pelo IPGMCC, "Staff" do Hospital Geral de Bonsucesso, VOT Imagem e Hospital São Lucas.

5. Interno e Monitor de Radiologia da FMT-CCBM - FESO, Estagiário em Radiologia do Hospital Santa Cruz/Beneficência Portuguesa de Niterói - IPGMCC.

6. Interna e Monitora Bolsista da Disciplina de Radiologia da FMT-CCBM - FESO.

7. Professor Titular do Curso de Pós-Graduação em Radiologia do IPGMCC, Professor Adjunto e Chefe do Serviço de Radiologia do Hospital Universitário Antônio Pedro da Universidade Federal Fluminense (HUAP-UFF), Chefe dos Centros de Diagnóstico por Imagem dos Hospitais de Clínicas de Niterói e da VOT Imagem.

Endereço para correspondência: Dr. Marcelo Souto Nacif. Rua Álvares de Azevedo, 130, ap. 704, bloco A, Icarai. Niterói, RJ, 24220-021. E-mail: marcelonacif30@hotmail.com e msnacif @yahoo.com.br

Recebido para publicação em 27/5/2003. Aceito, após revisão, em 22/12/2003. venosa pode ser realizada por veias pulmonares ou veias sistêmicas, como do sistema ázigos ou hemiázigos ${ }^{(1-3)}$.

Existem duas formas descritas: sequiestro intralobar (SIL) e seqüestro extralobar (SEL). Na primeira forma, a região pulmonar anômala está incorporada ao parênquima pulmonar normal, compartilhando o mesmo envoltório pleural visceral. Na segunda forma, o pulmão anômalo apresenta envoltório pleural próprio, geralmente em íntima proximidade com o pulmão normal, mas, eventualmente, abaixo do diafragma $^{(1,4-6)}$.

Sequiestro pulmonar é uma das anormalidades pulmonares congênitas mais comuns, em que a forma intralobar representa cerca de $75 \%$ dos $\operatorname{casos}^{(2,3,7)}$.

Neste trabalho relatamos um caso de seqüestro pulmonar intralobar, destacando os principais achados dos estudos de imagem.

\section{RELATO DO CASO}

Paciente do sexo feminino, 49 anos de idade, natural do Ceará, apresentando episódios de infecção pulmonar de repetição há cinco anos. Negava tabagismo. Em junho de 1999 teve novo quadro de infecção pulmonar aguda, com febre e tosse com escarro mucopurulento. Foi internada para melhor investigação diagnóstica.
Foi realizada telerradiografia do tórax em incidências póstero-anterior e perfil, que mostraram opacidade homogênea configurando massa no segmento basal posterior do pulmão direito (Figura 1). Foi feita angiotomografia helicoidal do tórax, em que foi possível observar a opacidade arredondada com suprimento sanguíneo proveniente da artéria aorta descendente (Figura 2). As reformatações 3D (Figura 3) reafirmaram os achados dos cortes axiais, confirmando o diagnóstico de seqüestro pulmonar intralobar.

A paciente foi submetida a segmentectomia da região acometida, evoluindo sem complicações e com melhora do quadro pulmonar. $\mathrm{O}$ exame da peça cirúrgica mostrou o sequiestro pulmonar com a cavidade preenchida por muco (Figura 4).

\section{DISCUSSÃO}

O SB é uma anomalia congênita do tecido pulmonar isolado da comunicação brônquica normal, e que recebe suprimento sanguíneo de um ramo arterial da circulação sistêmica.

Pertence a um espectro de anomalias denominadas malformações broncopulmonares e do trato digestivo anterior. Essas anomalias incluem grande variedade de defeitos: agenesia e hipoplasia do pulmão, $\mathrm{SB}$, suprimento arterial sistêmico aberrante 


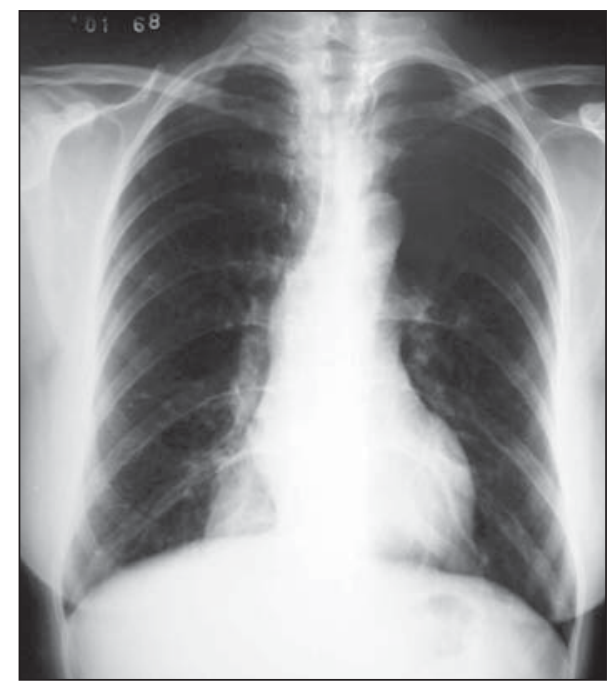

A

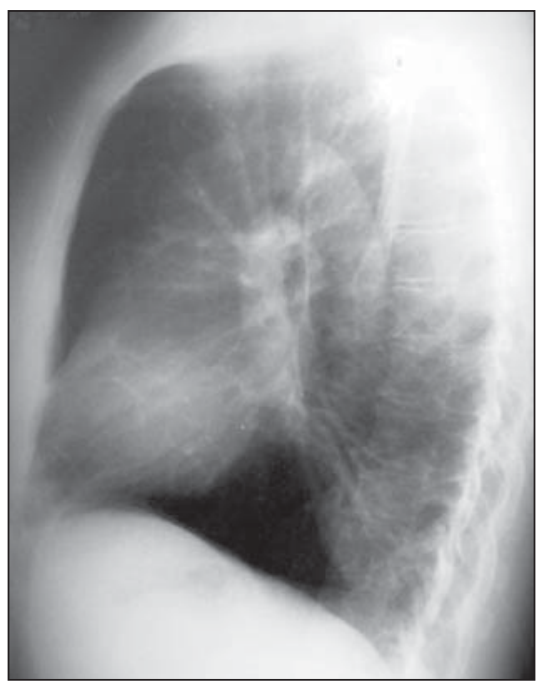

B

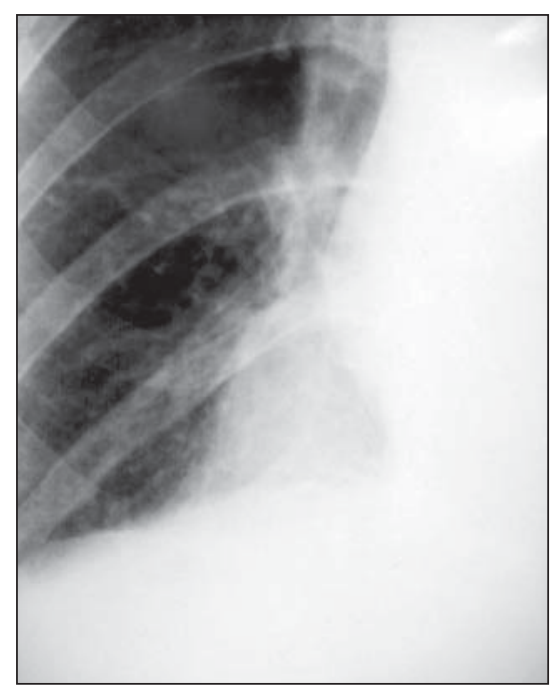

C

Figura 1. Telerradiografia de tórax em póstero-anterior (A), perfil (B) e localizada (C). Observar a opacidade homogênea configurando massa no segmento basal posterior do pulmão direito.

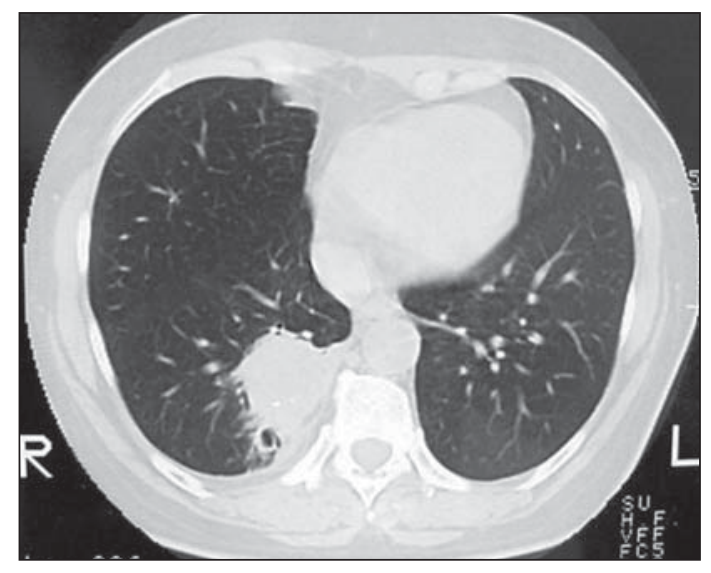

A

ao pulmão, malformação adenomatóide cística congênita, cistos broncogênicos, comunicação broncoesofágica e broncogástrica, restos da mucosa brônquica na parede esofágica, divertículos congênitos do trato gastrintestinal e dos brônquios, fístulas traqueoesofágicas, atresia do esôfago, cistos de duplicação do esôfago e cistos neuroentéricos ${ }^{(\mathbf{1 , 3}, 7)}$.

O SB é designado como intra ou extralobar, de acordo com a relação do tecido sequiestrado com o pulmão. O SIL é uma anomalia em que uma artéria sistêmica, originando-se normalmente da aorta torácica descendente $(73 \%)$ ou aorta abdominal $(20 \%)$, estende-se até um tecido pulmonar que não esteja ligado à arvore brônquica normal e recebe, por isso, denominação de seqüestrado. A drenagem venosa

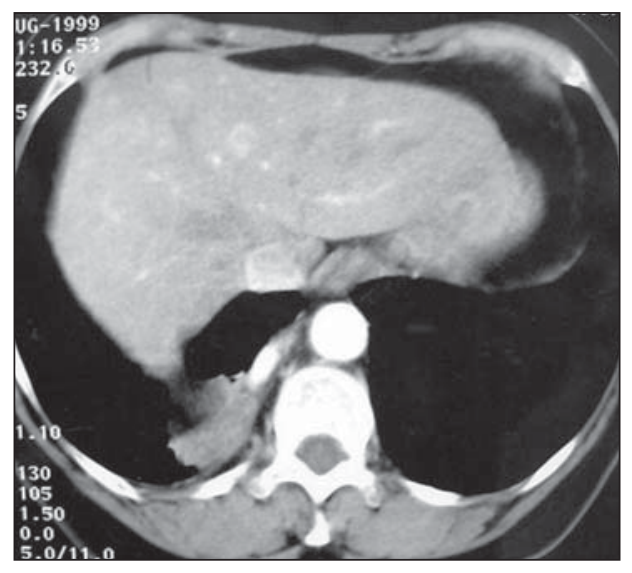

Figura 2. Tomografia computadorizada, janelas para parênquima (A) e para mediastino (B). Notar que existe um suprimento sanguíneo evidente, proveniente da artéria aorta descendente, dirigindo-se para a opacidade.
B

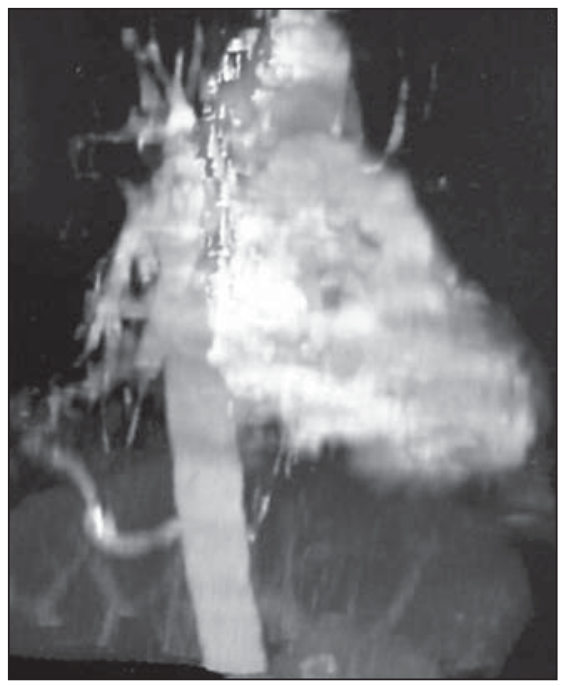

A

Figura 3. Angiotomografia computadorizada com reconstrução em 3D. Observar o suprimento sanguíneo mais bem visualizado. 


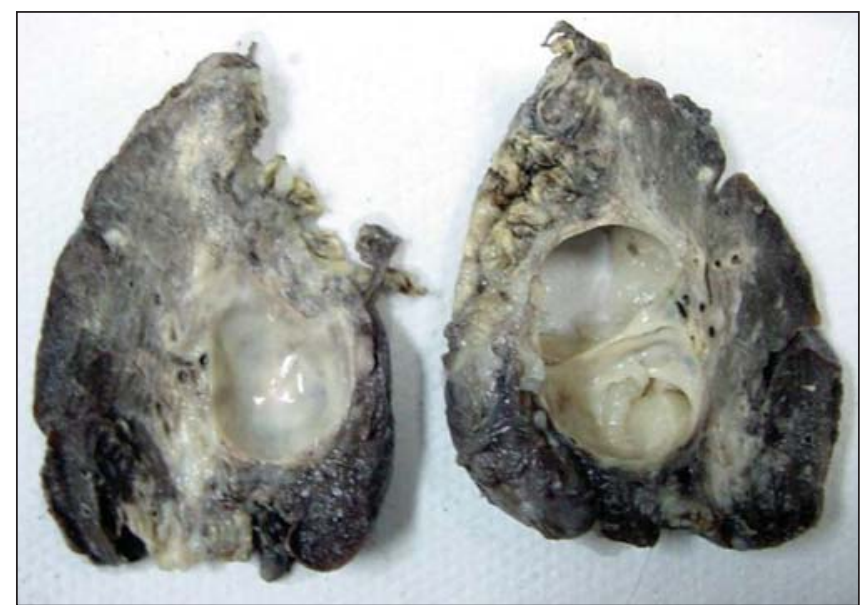

A

Figura 4. Peça cirúrgica demonstrando o seqüestro pulmonar intralobar com cavidade preenchida por muco.

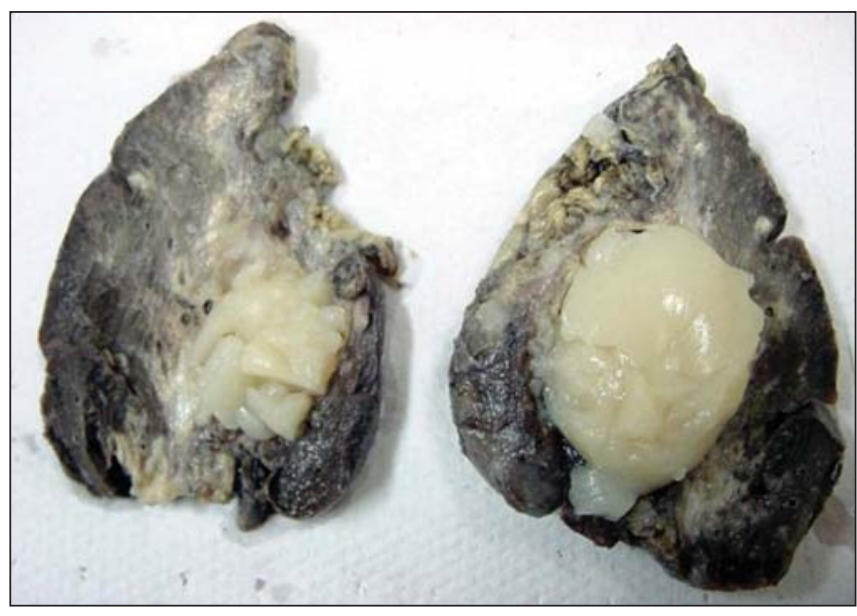

B é, geralmente, pelo sistema venoso pulmonar e menos freqüentemente pela circulação sistêmica, por meio das veias intercostal, ázigos ou hemiázigos, ou pela veia cava inferior ou superior. Em $98 \%$ dos casos o SIL ocorre num lobo pulmonar inferior, geralmente à esquerda. O diagnóstico geralmente é feito no início da vida adulta, quando o SIL se infecta e a maioria dos pacientes apresenta-se com pneumonia. $\mathrm{O}$ diagnóstico definitivo do SIL é feito por intermédio do estudo anatomopatológico, mas pode ser sugerido por métodos de imagem como ultra-sonografia (US) com Doppler colorido, tomografia computadorizada (TC) helicoidal, ressonância magnética (RM) e arteriografia ${ }^{(2,4,5)}$.

O SEL ocorre quando o tecido seqüestrado está contido em seu próprio revestimento pleural, podendo estar localizado entre o lobo inferior e o diafragma, no diafragma, no mediastino, no pericárdio, numa fissura, fixado à parede torácica ou sob o diafragma. O suprimento arterial é comumente da aorta abdominal ou de um de seus ramos, e a drenagem é via sistema venoso sistêmico pela veia cava inferior, veias ázigos ou hemiázigos e, ocasionalmente, pelo sistema porta ${ }^{(\mathbf{1 , 5})}$.

O diagnóstico definitivo depende da exata visualização dos vasos que nutrem e drenam o seqüestro, condição essencial para o planejamento cirúrgico ${ }^{(2,7)}$.
A apresentação radiográfica mais comum é de uma opacidade homogênea no segmento basal posterior do lobo inferior (geralmente à esquerda e em contigüidade com o hemidiafragma); menos comumente pode apresentar-se como massa cística com nível líquido único ou múltiplos ${ }^{(4)}$.

A US tem utilidade como método nãoinvasivo na avaliação de massas torácicas no período pré-natal e em recém-nascidos. Os SB manifestam-se como massas intratorácicas ecogênicas, sólidas, podendo mostrar áreas císticas, sendo mais bem avaliadas na ausência de parênquima pulmonar aerado interposto. O Doppler colorido ajuda na visualização da artéria anômala nutridora $^{(5,7)}$.

A arteriografia permite caracterizar bem as artérias anômalas, sua origem, número, curso, tamanho e drenagem venosa, informações valiosas no planejamento pré-operatório $^{(1,2)}$.

Os aspectos tomográficos são variados, podendo-se observar desde a presença de massa homogênea até formações císticas. Áreas de baixa atenuação podem ser explicadas pela presença de ventilação colateral e aprisionamento de ar. $\mathrm{O}$ achado de calcificações é bastante incomum. A TC pode demonstrar fidedignamente a origem e o curso dos vasos anômalos sistêmicos, principalmente após o advento dos equipamentos helicoidais. Em geral as imagens axiais são suficientes para estabelecer o diagnóstico, podendo-se também utilizar as reconstruções 3D dos grandes vasos, permitindo maior detalhamente de suas relações anatômicas ${ }^{(3,4)}$.

A RM permite excelente visualização dos vasos anômalos em múltiplos planos, muitas vezes dispensando a necessidade da angiografia. Além disso, pode demonstrar melhor as características da lesão pulmonar, como sendo cística, sólida, fluida, hemorrágica e/ou mucosa ${ }^{(4,5)}$.

\section{REFERÊNCIAS}

1. Coulier B, Mailleux P, Van Cutsem O, Bachez P, Mairesse M, Ledent C. Diagnosis of intralobar pulmonary sequestration using helical computed tomography angiography: apropos of 3 patients. JBRBTR 1999;82:6-10.

2. Grigoryants V, Sargent SK, Shorter NA. Extralobar pulmonary sequestration receiving its arterial supply from the innominate artery. Pediatr Radiol 2000;30:696-8.

3. Amitai M, Konen E, Rozenman J, Gerniak A. Preoperative evaluation of pulmonary sequestration by helical CT angiography. AJR 1996;167:1069-70.

4. Mooney DP, Sargent SK, Pluta D, Mazurek P. Spiral CT: use in the evaluation of chest masses in the critically ill neonate. Pediatr Radiol 1996;26:158.

5. Lange S, Walsh G. Radiology of chest diseases. 2nd ed. New York, NY: Thieme, 1998.

6. Freitas LO, Nacif MS. Radiologia prática para o estudante de medicina. Vol. II. Rio de Janeiro, RJ: Revinter, 2003.

7. Malheiros NR, Marchiori E, Miyagui T. Seqüestro pulmonar calcificado - relato de um caso. Radiol Bras 1996;29:357-60. 